Research Paper

\title{
Prognostic Significance of Preoperative Albumin to Alkaline Phosphatase Ratio in Patients with Glioblastoma
}

\author{
Junhong Li, M.D.; Mingrong Zuo, M.D.; Xingwang Zhou, M.D.; Yufan Xiang, M.D.; Shuxin Zhang, M.D.; \\ Wentao Feng, M.D.; Yanhui Liu, M.D., Ph.D. ${ }^{凶}$ \\ Department of Neurosurgery, West China Hospital of Sichuan University, Chengdu 610041, Sichuan Province, P.R. China. \\ $\triangle$ Corresponding author: Yanhui Liu, E-mail: yhliu2001@163.com.
}

(c) The author(s). This is an open access article distributed under the terms of the Creative Commons Attribution License (https://creativecommons.org/licenses/by/4.0/). See http://ivyspring.com/terms for full terms and conditions.

Received: 2021.04.21; Accepted: 2021.08.01; Published: 2021.08.13

\begin{abstract}
Objective: To explore the prognostic value of preoperative albumin to alkaline phosphatase ratio (AAPR) in patients with newly-diagnosed glioblastoma (GBM) and its association with clinical characteristics.

Patients and methods: A retrospective analysis was carried out on patients with newly diagnosed GBM who had undergone operation at the Department of Neurosurgery at West China Hospital between June 1st 2016 to December 31st 2018. X-tile software was applied to determine the optimal cut-off values for AAPR, neutrophil to lymphocyte ratio (NLR), and albumin. Cox regression analyses were applied to evaluate the prognostic value of AAPR in GBM. PSM analysis was conducted to verify the results.

Results: A total of 197 and 154 GBM patients were included in original cohort and PSM cohort respectively. The optimal cut-off value for AAPR, NLR, and albumin were $0.56,4.55$ and $42.2 \mathrm{~g} / \mathrm{L}$ respectively. High AAPR was only significantly related to longer overall survival $(O S)(p=0.010)$ in original cohort. In PSM cohort, no clinical variable was evidently related to the level of AAPR. AAPR was determined to be an independent prognostic indicator in both original cohort $(H R=0.599,95 \% \mathrm{Cl} 0.437-0.822, p=0.001)$ and PSM cohort $(\mathrm{HR}=0.649,95 \% \mathrm{Cl} 0.459-0.918, \mathrm{p}=0.015)$. Prognostic models including AAPR had better prognostic accuracy than that including albumin.

Conclusion: Preoperative AAPR was determined to be an independent risk factor of prognosis in newly-diagnosed GBM patients, and its prognostic ability was stronger than albumin. And PSM analysis also validated the results.
\end{abstract}

Key words: Albumin to alkaline phosphatase ratio; glioblastoma; prognosis; propensity score matching

\section{Introduction}

Glioblastoma (GBM) is the most lethal diffuse glioma with a median overall survival of 14-17 months and is characterized histologically by marked cellularity, prominent mitotic activity, abundant vascular proliferation and necrosis [1, 2]. GBM is recognized as grade IV tumor by the World Health Organization (WHO), constituting $45.2 \%$ of all malignant central nervous system (CNS) tumors and $80 \%$ of all primary malignant CNS tumors [3]. Based on the latest 2016 WHO classification of CNS tumors, GBMs are divided into three types, including IDHwildtype, IDH-mutant, and not otherwise specified (NOS) glioblastoma [4]. Surgery is the optimal choice in patients with suspected malignant glioma, and postoperative radiotherapy and chemotherapy are considered to be the first-line adjuvant treatments, which can help prolong lifetime to the maximum extent [1]. In recent years, the biological nature of GBM has been well comprehended by deep DNA and RNA sequencing, coupled with improved techniques that enable richer interrogation of the epigenome [5]. At the same time, cheaper and more convenient methods including neuroimaging, clinical examination, histopathology, etc. are also contributing to a well-rounded understanding of GBM.

Nowadays, cumulative evidence has indicated that some blood parameters are related to tumorigenesis and tumor progression [6-8]. Several biomarkers such as neutrophil-to-lymphocyte ratio 
(NLR), platelet-to-lymphocyte ratio (PLR), and fibrinogen are considered to be associated with clinical outcomes [9-12]. Albumin is one of the important compositions of the blood and constitutes $50 \%$ of the plasma. It is responsible for $75 \%$ of the plasma oncotic pressure and increases circulating blood volume. Albumin performs a variety of physiological functions, including ligand binding and drugs transport, free radical scavenging, anti-oxidant function, effect on vascular permeability, etc. [13]. The hydrolase enzyme alkaline phosphatase (ALP) is widely expressed in human tissues such as liver, bones and kidneys [14]. Similarly, it plays an important role in human physiological functions, including bone mineralization, vascular calcification, and regulations of immune system [15-17]. The albumin to alkaline phosphatase ratio (AAPR), based on serum albumin and alkaline phosphatase, is a useful prognostic indicator. It has been found to have significant impacts on survival in patients with liver cancer [18] and predictive ability on prognosis in various tumors [19-22].

The relationship between AAPR and GBM has not been explored so far. In the current study, we attempted to figure out the prognostic value of preoperative AAPR in patients with GBM, and investigated its association with clinical characteristics.

\section{Patients and Methods}

\section{Patient Population}

A retrospective chart review was carried out on patients with newly diagnosed GBM who had undergone an operation at the Department of Neurosurgery at West China Hospital between June $1^{\text {st }} 2016$ to December 31 ${ }^{\text {st }} 2018$. All patients underwent a craniotomy on GBM with gross total resection (GTR) or subtotal resection (STR). These patients were followed up until June $31^{\text {st }} 2020$. The pathological diagnoses were based on 2016 WHO classification of CNS tumors.

The inclusion criteria included: 1) older than 18-year-old; 2) underwent resection of GBM by craniotomy with GTR or STR; 3) the pathological diagnoses were based on the latest 2016 WHO classification; 4) intact baseline clinical data; 5) intact preoperative MRI imaging data and postoperative imaging data including MRI and CT within 72 hours after operation; 6) no adjuvant therapy like chemotherapy or radiotherapy before operation; 7) no history of diseases dramatically affecting peripheral blood cells. The exclusion criteria were: 1) younger than 18-year-old; 2) biopsy only; 3 ) absence of definite pathological diagnosis; 4) incomplete baseline clinical data; 5) absence of preoperative MRI imaging data; 6) receiving adjuvant therapy before operation; 7) presence of history of liver diseases, bone diseases, urological diseases, or infectious diseases shortly before surgery.

\section{Parameters Assessment}

Medical records were surveyed and the following clinical data were retrieved: gender, age at operation, preoperative Karnofsky performance status, presence of preoperative seizures, locations and hemisphere of tumors, pathological diagnoses and useful biomarkers. Ki-67 index was tested by immunohistochemistry (IHC), and IDH-1 mutations were determined by both IHC and molecular testing. Routine blood tests were performed within 3 days prior to surgery and relevant data was recorded. The APPR was defined as the ratio between the serum albumin concentration $(\mathrm{g} / \mathrm{L})$ and the alkaline phosphatase $(\mathrm{U} / \mathrm{L})$, while the NLR was defined by dividing the neutrophil $\left(\times 10^{9} / \mathrm{L}\right)$ count by the lymphocyte count $\left(\times 10^{9} / \mathrm{L}\right)$.

Postoperative adjuvant therapies and survival conditions were collected mainly through periodical telephone interview and outpatient follow-up. Patients were routinely followed up every 3 months for the first year, and every 6 months thereafter. Overall survival was defined as the duration from the date of surgery to death or the last follow up. All clinical assessments were performed by two independent qualified neurosurgeons.

\section{Statistical Analysis}

SPSS 22.0 (IBM Co., Armonk, NY, USA) was used for all statistical analyses. X-tile software was applied to determine the optimal cut-off values for AAPR, NLR, and albumin [23]. The associations between APPR and clinical variables were tested by chi-square test, Mann-Whitney test, or one-way ANOVA (one-factor analysis of variance). The Cox regression analyses were used to determine the influence of risk factors for overall survival in GBM patients. In Cox regression analyses, a univariate Cox regression was firstly conducted to evaluate clinical variables, then variables with $p$ value $<0.1$ were included into a backward stepwise multivariate Cox regression for further assessment. R software (version 3.6.3, http://www.r-project.org/) was applied to calculate and compare Harrell concordance index (C-index) and Akaike information criterion (AIC) of prognostic models. A smaller AIC value and/or a larger C-index represented a greater predictive accuracy. A two-sided p-value $<0.05$ referred as statistically significant difference. 
Propensity score matching (PSM) analysis was introduced in the current study to adjust for confounding variables and validate the results of the original cohort. The potential confounding covariables included age at diagnosis, gender, preoperative seizures, KPS, hemisphere, location, Ki-67 index, and IDH-1 status. These patients were matched 1:1 using the nearest-neighbor algorithm with a caliper width of 0.2 and without replacement.

\section{Ethics}

This study was approved by the Ethical Committee of Sichuan University and conducted according to the principles expressed in the Declaration of Helsinki, and all patients were informed and signed their informed consent to use their data for research purposes.

\section{Results}

\section{Baseline Characteristics}

The screening process was listed in Figure 1. The original cohort was constituted by a total of 197 patients with craniotomy for histologically-proven glioblastoma (Table 1). There were 120 (60.9\%) males and $77(39.1 \%)$ females, with a mean age of 54.58 \pm 0.975 years (median 55 , range $20-85$ years). The mean follow-up period was $467.06 \pm 24.57$ days (median 357, range 35-1611 days). In terms of tumor-related seizures, $32(16.2 \%)$ patients were diagnosed with preoperative seizures. A preoperative KPS score $>80$ was recorded in $61(31.0 \%)$ patients and vice versa. For location of GBMs, 95 (48.2\%) were located at left hemisphere, $91(46.2 \%)$ at right hemisphere, and 11 $(5.6 \%)$ at midline regions or invading bilateral brain tissues. These tumors were distributed in frontal lobe (22.84\%), temporal lobe (14.21\%), parietal lobe $(4.1 \%)$, and occipital lobe $(1.0 \%)$, and the rest were involved in multiple regions $(57.9 \%)$. As for postoperative adjuvant therapy, $69(35.0 \%)$ patients received both chemotherapy and radiotherapy, $59(30.0 \%)$ patients received one of the two treatments, while other 69 (35.0\%) patients didn't receive any kind of adjuvant therapy. Specific tumor-related biomarkers were recorded; a total of 104 (52.8\%) patients had ki-67 index $<30 \%$, while 31 patients were tested as IDH-1 mutation. The optimal cut-off value for AAPR, NLR, and albumin were $0.56,4.55$, and $42.2 \mathrm{~g} / \mathrm{L}$ calculating by the X-tile software, respectively (Figure 2). An AAPR $>0.56$ was found in $116(58.9 \%)$ patients, and an NLR $>4.55$ was shown in $45(22.8 \%)$ patients.

After 1:1 PSM stratified by the optimal cut-off value of AAPR, a total of 154 patients with 77 AAPR $>0.56$ and 77 AAPR $\leq 0.56$ were included in PSM cohort. This cohort had a high similarity to original cohort in constitution.

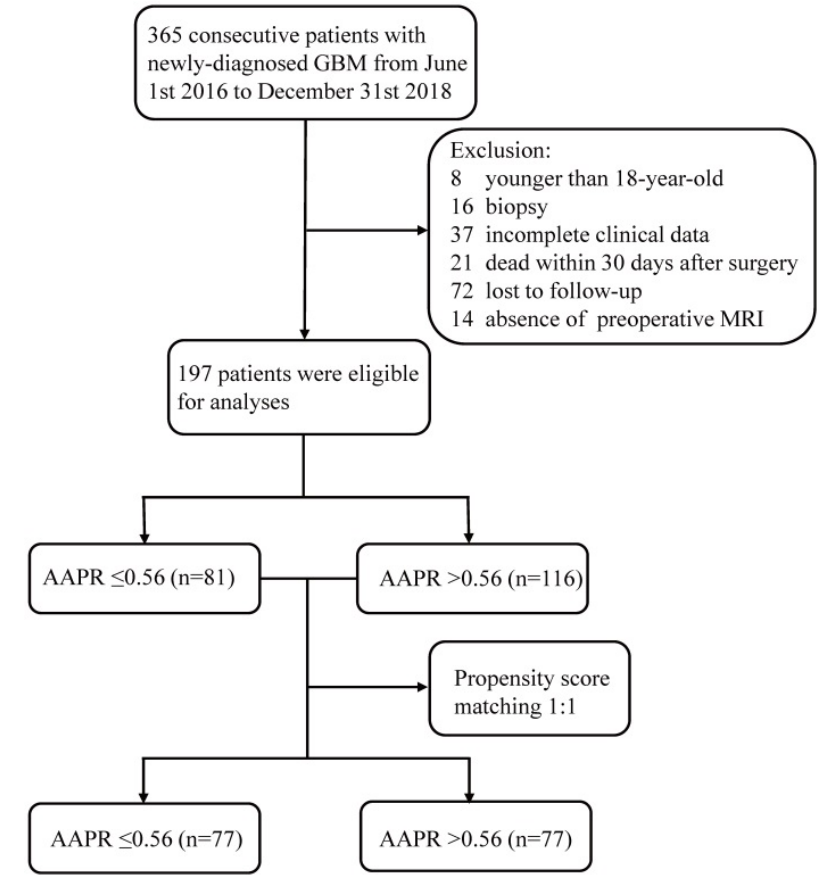

Figure 1. Flow chart of the current study. Abbreviation: GBM, glioblastoma; MRI, magnetic resonance imaging; AAPR, albumin to alkaline phosphatase ratio.

Table 1. Baseline clinical characteristics of GBM patients in original cohort and PSM cohort

\begin{tabular}{|c|c|c|}
\hline Clinical Characteristic & Original cohort & PSM cohort \\
\hline Number of patients & $197(100)$ & $154(100)$ \\
\hline \multicolumn{3}{|l|}{ Follow-up period } \\
\hline Mean \pm SD (day) & $467.06 \pm 344.83$ & $443.62 \pm 322.78$ \\
\hline Median (range) & 357 (35-1611) & 337 (38-1611) \\
\hline \multicolumn{3}{|l|}{ Age at operation } \\
\hline Mean \pm SD (year) & $54.58 \pm 13.68$ & $55.46 \pm 13.11$ \\
\hline Median (range) & $55(20-85)$ & $56(20-85)$ \\
\hline \multicolumn{3}{|l|}{ Gender } \\
\hline Male & $120(60.9)$ & $100(64.9)$ \\
\hline Female & 77 (39.1) & $54(35.1)$ \\
\hline \multicolumn{3}{|l|}{ Preoperative seizures } \\
\hline Yes & $32(16.2)$ & $26(16.9)$ \\
\hline No & $165(83.8)$ & $128(83.1)$ \\
\hline \multicolumn{3}{|l|}{ KPS } \\
\hline$>80$ & $61(31.0)$ & $45(29.2)$ \\
\hline$\leq 80$ & $136(69.0)$ & $109(70.8)$ \\
\hline \multicolumn{3}{|l|}{ Hemisphere } \\
\hline Left & $95(48.2)$ & $73(47.4)$ \\
\hline Right & $91(46.2)$ & $71(46.1)$ \\
\hline Midline or bilateral & $11(5.6)$ & $10(6.5)$ \\
\hline \multicolumn{3}{|l|}{ Location } \\
\hline Frontal lobe & $45(22.8)$ & $36(23.4)$ \\
\hline Temporal lobe & $28(14.2)$ & $24(15.6)$ \\
\hline Parietal lobe & $8(4.1)$ & $4(2.6)$ \\
\hline Occipital lobe & $2(1.0)$ & $1(0.6)$ \\
\hline Other locations & $114(57.9)$ & $89(57.8)$ \\
\hline \multicolumn{3}{|l|}{ Adjuvant therapy } \\
\hline Chemotherapy and radiotherapy & $69(35.0)$ & $56(36.4)$ \\
\hline Chemotherapy or radiotherapy & $59(30.0)$ & $46(29.9)$ \\
\hline None & $69(35.0)$ & $52(33.7)$ \\
\hline \multicolumn{3}{|l|}{ Ki-67 } \\
\hline$\geq 30 \%$ & $93(47.2)$ & $76(49.4)$ \\
\hline$<30 \%$ & $104(52.8)$ & $78(50.6)$ \\
\hline \multicolumn{3}{|l|}{ IDH-1 } \\
\hline Mutant & $31(15.7)$ & $21(13.6)$ \\
\hline Wildtype & $166(84.3)$ & $133(86.4)$ \\
\hline
\end{tabular}




\begin{tabular}{lll}
\hline Clinical Characteristic & Original cohort & PSM cohort \\
\hline$>4.55$ & $45(22.8)$ & $38(24.7)$ \\
$\leq 4.55$ & $152(77.2)$ & $116(75.3)$ \\
$\begin{array}{l}\text { Albumin }(\mathrm{g} / \mathrm{L}) \\
>42.2\end{array}$ & $105(53.3)$ & $80(51.9)$ \\
$\leq 42.2$ & $92(46.7)$ & $74(48.1)$ \\
AAPR & & \\
$>0.56$ & $116(58.9)$ & $77(50.0)$ \\
$\leq 0.56$ & $81(41.1)$ & $77(50.0)$ \\
\hline
\end{tabular}

Data are expressed as $n(\%)$, mean $\pm S D$, or median (range);

Abbreviations: GBM, glioblastoma; PSM, propensity score matching; KPS,

Karnofsky performance status; IDH-1, Isocitrate dehydrogenase-1; NLR, neutrophil

to lymphocyte ratio; AAPR, albumin to alkaline phosphatase ratio.

\section{Relationships between AAPR and Clinical Variables}

The relationships between AAPR and other clinical variables were shown in Table 2. In original cohort, high AAPR was only significantly related to

OS $(p=0.010)$, and there was no significant association between AAPR and other clinical variables. In PSM cohort, however, there were not any clinical variables evidently related to the level of AAPR.

\section{Prognostic value of AAPR}

Univariate and multivariate Cox regressions were performed to further determine the prognostic significance of AAPR. As shown in Table 3, in original cohort, univariate Cox regression indicated high AAPR (HR 0.596, 95\%CI 0.439-0.810, $\mathrm{p}=0.001$ ) and high albumin (HR 0.617, 95\% CI 0.454-0.837, $\mathrm{p}=0.002$ ) were significantly associated with better OS, while high NLR was related to poor prognosis (HR 1.490, 95\% CI 1.045-2.125, $\mathrm{p}=0.028)$. Other significant variables included age, gender, KPS, hemisphere, adjuvant therapy, ki-67 index, and IDH-1 status.
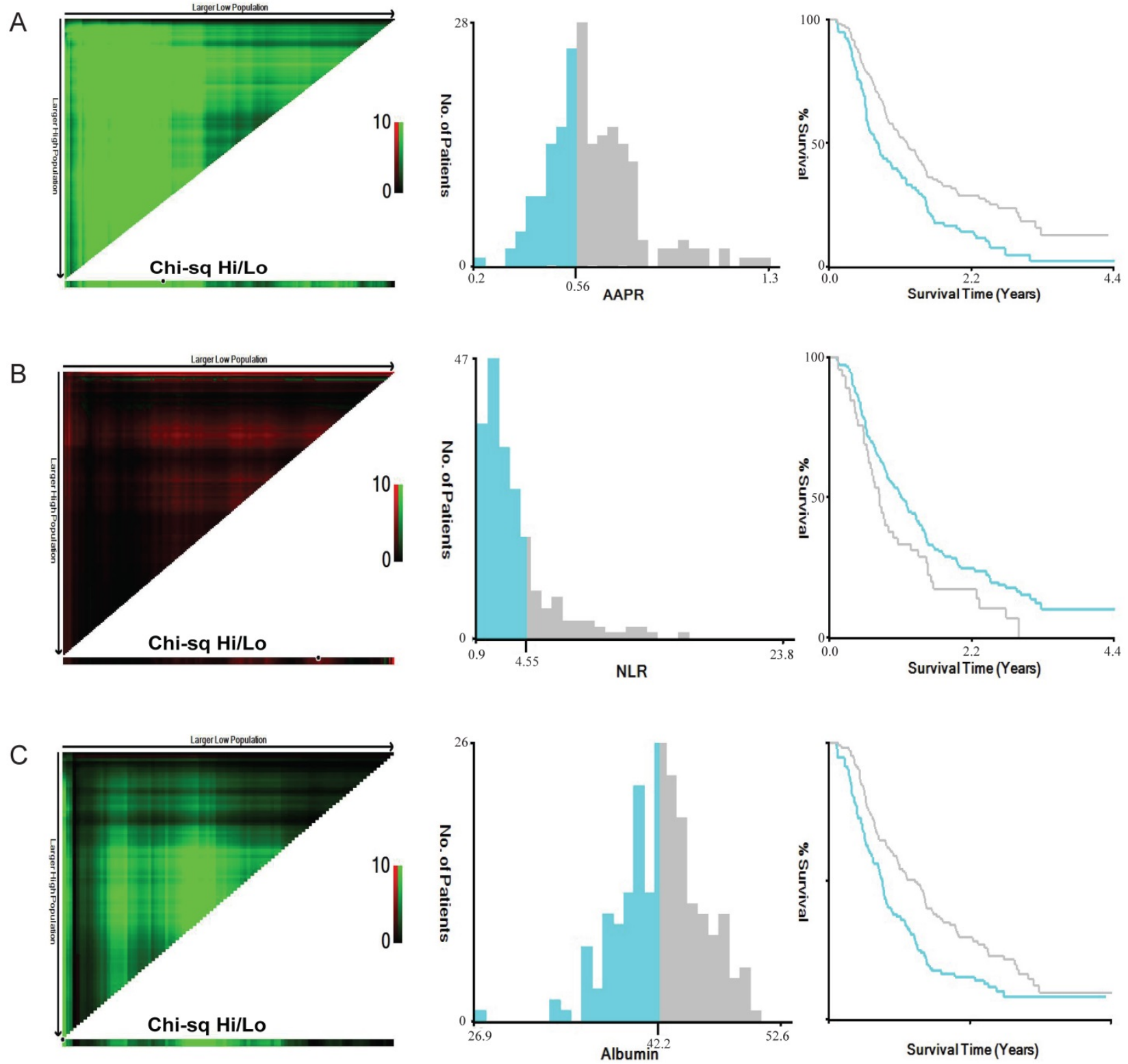

Figure 2. Calculation of optimal cut-off values of AAPR (A), NLR (B) and albumin (C) by X-tile software. Abbreviation: AAPR, albumin to alkaline phosphatase ratio; NLR, neutrophil to lymphocyte ratio. 
Table 2. Relationship between AAPR and clinical characteristics in original cohort and PSM cohort

\begin{tabular}{|c|c|c|c|c|c|c|}
\hline \multirow[t]{2}{*}{ Clinical Characteristic } & \multicolumn{3}{|l|}{ Original cohort } & \multicolumn{3}{|l|}{ PSM cohort } \\
\hline & AAPR $\leq 0.56(n=81)$ & AAPR $>0.56(n=116)$ & $\mathrm{p}$ value & AAPR $\leq 0.56(n=77)$ & AAPR $>0.56(n=77)$ & $\mathrm{p}$ value \\
\hline Age at operation & $53.65 \pm 13.78$ & $55.93 \pm 13.51$ & 0.166 & $55.12 \pm 13.32$ & $55.81 \pm 12.98$ & 0.884 \\
\hline OS & $277(35-1611)$ & $421(38-1575)$ & 0.010 & $286(43-1611)$ & $384(38-1575)$ & 0.140 \\
\hline \multicolumn{7}{|l|}{ Gender } \\
\hline Male & $52(64.2)$ & $68(58.6)$ & 0.431 & $49(63.6)$ & $51(66.2)$ & 0.736 \\
\hline Female & $29(35.8)$ & $48(41.4)$ & & $28(36.4)$ & $26(33.8)$ & \\
\hline \multicolumn{7}{|l|}{ Preoperative seizures } \\
\hline Yes & $15(18.5)$ & $17(14.7)$ & 0.471 & $14(18.2)$ & $12(15.6)$ & 0.668 \\
\hline No & $66(81.5)$ & $99(85.3)$ & & $63(81.8)$ & $65(84.4)$ & \\
\hline \multicolumn{7}{|l|}{ KPS } \\
\hline$>80$ & $24(29.6)$ & 37 (31.9) & 0.736 & $23(29.9)$ & $22(28.6)$ & 0.860 \\
\hline$\leq 80$ & $57(70.4)$ & $79(68.1)$ & & $54(70.1)$ & $55(71.4)$ & \\
\hline \multicolumn{7}{|l|}{ Hemisphere } \\
\hline Left & $37(45.7)$ & $58(50.0)$ & 0.366 & $35(45.5)$ & $36(46.7)$ & 0.630 \\
\hline Right & $36(44.4)$ & $55(47.4)$ & & $35(45.5)$ & $38(49.4)$ & \\
\hline Midline or bilateral & $8(9.9)$ & $3(2.6)$ & & $7(9.0)$ & $3(3.9)$ & \\
\hline \multicolumn{7}{|l|}{ Location } \\
\hline Frontal lobe & $19(23.4)$ & $26(22.4)$ & 0.511 & $18(23.4)$ & $18(23.4)$ & 0.785 \\
\hline Temporal lobe & $16(19.8)$ & $12(10.3)$ & & 15 (19.5) & $9(11.7)$ & \\
\hline Parietal lobe & $1(1.2)$ & $7(6.0)$ & & $0(0.0)$ & $4(5.2)$ & \\
\hline Occipital lobe & $0(0.0)$ & $2(1.7)$ & & $0(0.0)$ & $1(1.3)$ & \\
\hline Other regions & 45 (55.6) & 69 (59.5) & & $44(57.1)$ & $45(58.4)$ & \\
\hline \multicolumn{7}{|l|}{ Ki-67 } \\
\hline$\geq 30 \%$ & $42(51.9)$ & $51(44.0)$ & 0.276 & 39 (50.6) & 37 (48.1) & 0.748 \\
\hline$<30 \%$ & $39(48.1)$ & $65(56.0)$ & & $38(49.4)$ & $40(51.9)$ & \\
\hline \multicolumn{7}{|l|}{ IDH-1 } \\
\hline Mutant & $9(11.1)$ & $22(19.0)$ & 0.137 & $9(11.7)$ & $12(15.6)$ & 0.483 \\
\hline Wildtype & $72(88.9)$ & $94(81.0)$ & & $68(88.3)$ & $65(84.4)$ & \\
\hline
\end{tabular}

Data are expressed as $\mathrm{n}(\%)$, mean $\pm \mathrm{SD}$, or median (range).

Significant findings are expressed in bold and italic.

Abbreviations: AAPR, albumin to alkaline phosphatase ratio; PSM, propensity score matching; KPS, Karnofsky performance status; IDH-1, isocitrate dehydrogenase-1; SD,

standard deviation.

Table 3. Univariate and multivariate Cox regression for risk factors predictive of GBM in original cohort

\begin{tabular}{|c|c|c|c|c|c|c|c|c|c|c|c|c|}
\hline & \multicolumn{4}{|c|}{ Univariate Analysis } & \multicolumn{4}{|c|}{ Multivariate Analysis (AAPR) } & \multicolumn{4}{|c|}{ Multivariate Analysis (Albumin) } \\
\hline & HR & LL & UL & $p$ value & HR & LL & UL & $\mathrm{p}$ value & HR & LL & UL & $\mathrm{p}$ value \\
\hline \multicolumn{13}{|l|}{ Age at operation } \\
\hline$\geq 55$ & 1 & & & & 1 & & & & 1 & & & \\
\hline$<55$ & 0.566 & 0.416 & 0.771 & $<0.001$ & 0.706 & 0.507 & 0.983 & 0.040 & 0.772 & 0.547 & 1.088 & 0.140 \\
\hline \multicolumn{13}{|l|}{ Gender } \\
\hline Male & 1 & & & & 1.000 & & & & 1.000 & & & \\
\hline Female & 0.670 & 0.488 & 0.919 & 0.013 & 0.663 & 0.472 & 0.931 & 0.018 & 0.671 & 0.480 & 0.937 & 0.019 \\
\hline \multicolumn{13}{|l|}{ KPS } \\
\hline$>80$ & 1 & & & & & & & & & & & \\
\hline$\leq 80$ & 1.233 & 0.884 & 1.721 & 0.218 & & & & & & & & \\
\hline \multicolumn{13}{|l|}{ Hemisphere } \\
\hline Midline or bilateral & 1 & & & & 1 & & & & 1 & & & \\
\hline Right & 0.502 & 0.259 & 0.974 & 0.041 & 0.767 & 0.387 & 1.521 & 0.448 & 0.748 & 0.378 & 1.477 & 0.403 \\
\hline Left & 0.395 & 0.203 & 0.769 & 0.006 & 0.511 & 0.256 & 1.021 & 0.057 & 0.487 & 0.245 & 0.967 & 0.040 \\
\hline \multicolumn{13}{|l|}{ Location } \\
\hline Frontal lobe & 1 & & & & & & & & & & & \\
\hline Temporal lobe & 1.639 & 0.983 & 2.731 & 0.058 & & & & & & & & \\
\hline Parietal lobe & 1.005 & 0.422 & 2.395 & 0.991 & & & & & & & & \\
\hline Occipital lobe & 0.383 & 0.052 & 2.813 & 0.346 & & & & & & & & \\
\hline Other regions & 1.319 & 0.896 & 1.943 & 0.160 & & & & & & & & \\
\hline \multicolumn{13}{|l|}{ Pre-operative seizures } \\
\hline No & 1 & & & & & & & & & & & \\
\hline Yes & 1.242 & 0.836 & 1.845 & 0.284 & & & & & & & & \\
\hline \multicolumn{13}{|l|}{ Adjuvant therapy } \\
\hline Chemotherapy and radiotherapy & 1 & & & & 1 & & & & 1 & & & \\
\hline Chemotherapy or radiotherapy & 2.591 & 1.745 & 3.847 & $<0.001$ & 3.016 & 1.998 & 4.553 & $<0.001$ & 3.205 & 2.124 & 4.835 & $<0.001$ \\
\hline None & 3.506 & 2.387 & 5.148 & $<0.001$ & 4.595 & 2.991 & 7.059 & $<0.001$ & 4.492 & 2.951 & 6.838 & $<0.001$ \\
\hline \multicolumn{13}{|l|}{ Ki67 } \\
\hline$\geq 30 \%$ & 1 & & & & 1 & & & & 1 & & & \\
\hline$<30 \%$ & 0.736 & 0.544 & 0.997 & 0.048 & 0.638 & 0.462 & 0.882 & 0.007 & 0.681 & 0.496 & 0.936 & 0.018 \\
\hline
\end{tabular}




\begin{tabular}{|c|c|c|c|c|c|c|c|c|c|c|c|c|}
\hline & \multicolumn{4}{|c|}{ Univariate Analysis } & \multicolumn{4}{|c|}{ Multivariate Analysis (AAPR) } & \multicolumn{4}{|c|}{ Multivariate Analysis (Albumin) } \\
\hline & $\overline{\mathrm{HR}}$ & LL & UL & $p$ value & HR & LL & UL & $p$ value & HR & LL & UL & $\mathrm{p}$ value \\
\hline \multicolumn{13}{|l|}{ IDH-1 } \\
\hline Positive & 1 & & & & 1 & & & & 1 & & & \\
\hline Negative & 2.765 & 1.665 & 4.590 & $<0.001$ & 2.055 & 1.209 & 3.492 & 0.008 & 2.369 & 1.396 & 4.021 & 0.001 \\
\hline \multicolumn{13}{|l|}{ NLR } \\
\hline Low & 1 & & & & 1 & & & & 1 & & & \\
\hline High & 1.490 & 1.045 & 2.125 & 0.028 & 1.028 & 0.706 & 1.498 & 0.884 & 0.985 & 0.672 & 1.444 & 0.938 \\
\hline \multicolumn{13}{|l|}{ AAPR } \\
\hline Low & 1 & & & & 1 & & & & & & & \\
\hline High & 0.596 & 0.439 & 0.810 & 0.001 & 0.599 & 0.437 & 0.822 & 0.001 & & & & \\
\hline \multicolumn{13}{|l|}{ Albumin } \\
\hline Low & 1 & & & & & & & & 1 & & & \\
\hline High & 0.617 & 0.454 & 0.837 & 0.002 & & & & & 0.670 & 0.484 & 0.929 & 0.016 \\
\hline
\end{tabular}

Significant findings are expressed in bold and italic.

Abbreviations: GBM, glioblastoma; HR, hazard ratio; CI, confidence interval; LL, lower limit; UL, upper limit; KPS, Karnofsky performance status; IDH-1, Isocitrate dehydrogenase-1; NLR, neutrophil to lymphocyte ratio; AAPR, albumin to alkaline phosphatase ratio.

Variables with a $\mathrm{p}$ value $<0.1$ was included in multivariate Cox regression. AAPR ( $\mathrm{HR}=0.599,95 \% \mathrm{CI}$ 0.437-0.822, $\mathrm{p}=0.001)$ and albumin $(\mathrm{HR}=0.670,95 \% \mathrm{CI}$ 0.484-0.929, $\mathrm{p}=0.016$ ) were proved to be independent risk factors of OS. Other independent risk factors included age, gender, adjuvant therapy, and ki-67 index, while hemisphere and NLR were not independent prognostic factors.

As regard to PSM cohort (Table 4), independent prognostic indicators from multivariate Cox regression included adjuvant therapy, IDH-1 status, AAPR, and albumin.

\section{Comparison of prognostic ability between AAPR and albumin}

To further compare the prognostic predictive ability of AAPR and albumin, prognostic models were established by using the two markers combined with other independent variables. In original cohort (Table 5), C-index and AIC were calculated by R software and the results indicated that model AAPR (C-index/AIC, 0.721/1452.24) was superior to model albumin (C-index/AIC, 0.715/1455.95). As the same, in PSM cohort (Table 6), model AAPR (C-index/AIC, $0.698 / 1091.43$ ) had advantage over model albumin (C-index/ AIC, 0.695/1094.86) in predictive accuracy.

\section{Discussion}

Peripheral blood markers were widely used in monitoring tumor progression and predicting prognosis of tumor patients. In the current study, we found higher AAPR was associated with better OS in patients with GBM. Multivariate analysis indicated AAPR as an independent risk factor for OS. And PSM analysis also verified the results.

The results are consistent with the conclusions of other researches about AAPR to some extent. Previous researches report that AAPR is evidently related to the clinical outcome of solid tumors, namely higher AAPR is significantly associated with better outcomes in survival or therapy of the tumors, such as upper tract urothelial carcinoma, cholangiocarcinoma, non-small-cell lung cancer, hepatocellular carcinoma, nasopharyngeal carcinoma, pancreatic ductal adenocarcinoma, etc. [18-20, 22, 24, 25]. Cut-off values of these studies were in the range of 0.36-0.84, and the cut-off value of AAPR in our research was 0.56. Due to large sample size, results of these different tumor researches are considered reliable.

Albumin is an important nutritional indicator and also associated with the process of systemic inflammation [26, 27]. Previous researches indicate albumin served as a prognostic predictor in various kind of tumors, including oral cavity squamous cell carcinoma, non-small cell lung cancer, advanced esophageal cancer, etc. [28-30]. Higher albumin level is often related to better clinical outcome. And to enhance the prediction, it is also combined with other blood biomarkers like Glasgow Prognostic Score (GPS) and Controlling Nutritional Status Score (CONUT) [31, 32]. In view of the origin of ALP, the correlations between ALP and prognosis of tumor were firstly reported in osteosarcoma, advanced prostate cancer, and hepatocellular carcinoma [33-35]. Then, studies of other tumors have found higher peripheral blood APL indicating shorter overall survival or progression-free survival [36-38]. This may explain why albumin to alkaline phosphatase ratio has a predictive ability of prognosis of tumors, but the underlying mechanism remains unclear.

Another hot blood biomarker NLR was introduced in our study to serve as a reference. As a marker of systemic inflammation, NLR is supposed to have predictive ability of prognosis in tumor patients based on the theory that inflammatory response plays an important role in tumor development and progression [11]. Most studies indicate that high NLR is related to shorter OS or PFS $[9,10,39,40]$. In the research of NLR and glioma, the situation becomes complicated. Majority of studies show that high NLR 
is significantly related to bad prognosis in Our study indicated that NLR was not an multivariate Cox regression analyses [41-44]. Conversely, some researches are unable to show that high NLR predicts shorter survival in GBM $[45,46]$. independent prognostic factor for GBM (multivariate Cox regression, HR 1.015, 95\% CI 0.696-1.481, $\mathrm{p}=0.937)$.

Table 4. Univariate and multivariate Cox regression for risk factors predictive of GBM in PSM cohort

\begin{tabular}{|c|c|c|c|c|c|c|c|c|c|c|c|c|}
\hline & \multicolumn{4}{|c|}{ Univariate Analysis } & \multicolumn{4}{|c|}{ Multivariate Analysis (AAPR) } & \multicolumn{4}{|c|}{ Multivariate Analysis (Albumin) } \\
\hline & \multirow[t]{2}{*}{ HR } & \multicolumn{2}{|c|}{$95 \% \mathrm{CI}$} & \multirow[t]{2}{*}{$\mathrm{p}$ value } & \multirow[t]{2}{*}{ HR } & \multicolumn{2}{|c|}{$95 \% \mathrm{CI}$} & \multirow[t]{2}{*}{$\mathrm{p}$ value } & \multirow[t]{2}{*}{ HR } & \multicolumn{2}{|c|}{$95 \% \mathrm{CI}$} & \multirow[t]{2}{*}{$\mathrm{p}$ value } \\
\hline & & LL & UL & & & LL & UL & & & LL & UL & \\
\hline \multicolumn{13}{|l|}{ Age at operation } \\
\hline$\geq 55$ & 1 & & & & 1 & & & & 1 & & & \\
\hline$<55$ & 0.675 & 0.477 & 0.954 & 0.025 & 0.878 & 0.613 & 1.258 & 0.479 & 0.984 & 0.682 & 1.420 & 0.932 \\
\hline \multicolumn{13}{|l|}{ Gender } \\
\hline Male & 1 & & & & & & & & & & & \\
\hline Female & 0.756 & 0.524 & 1.091 & 0.135 & & & & & & & & \\
\hline \multicolumn{13}{|l|}{ KPS } \\
\hline$>80$ & 1 & & & & & & & & & & & \\
\hline$\leq 80$ & 1.013 & 0.699 & 1.468 & 0.947 & & & & & & & & \\
\hline \multicolumn{13}{|l|}{ Hemisphere } \\
\hline Midline or bilateral & 1 & & & & & & & & & & & \\
\hline Right & 0.580 & 0.287 & 1.172 & 0.129 & & & & & & & & \\
\hline Left & 0.458 & 0.226 & 0.931 & 0.031 & & & & & & & & \\
\hline \multicolumn{13}{|l|}{ Location } \\
\hline Frontal lobe & 1 & & & & & & & & & & & \\
\hline Temporal lobe & 1.404 & 0.804 & 2.453 & 0.233 & & & & & & & & \\
\hline Parietal lobe & 0.919 & 0.280 & 3.024 & 0.890 & & & & & & & & \\
\hline \multicolumn{13}{|l|}{ Occipital lobe } \\
\hline Other regions & 1.248 & 0.813 & 1.917 & 0.311 & & & & & & & & \\
\hline \multicolumn{13}{|l|}{ Pre-operative seizures } \\
\hline No & 1 & & & & & & & & & & & \\
\hline Yes & 1.104 & 0.709 & 1.719 & 0.660 & & & & & & & & \\
\hline \multicolumn{13}{|l|}{ Adjuvant therapy } \\
\hline Chemotherapy and radiotherapy & 1 & & & & 1 & & & & 1 & & & \\
\hline Chemotherapy or radiotherapy & 2.809 & 1.803 & 4.375 & $<0.001$ & 2.866 & 1.834 & 4.478 & $<0.001$ & 3.119 & 1.986 & 4.899 & $<0.001$ \\
\hline None & 4.085 & 2.632 & 6.339 & $<0.001$ & 4.062 & 2.605 & 6.335 & $<0.001$ & 3.754 & 2.410 & 5.849 & $<0.001$ \\
\hline \multicolumn{13}{|l|}{ Ki67 } \\
\hline$\geq 30 \%$ & 1 & & & & & & & & & & & \\
\hline$<30 \%$ & 0.813 & 0.579 & 1.143 & 0.235 & & & & & & & & \\
\hline IDH-1 & & & & & & & & & & & & \\
\hline Positive & 1 & & & & 1 & & & & 1 & & & \\
\hline Negative & 2.688 & 1.474 & 4.901 & 0.001 & 2.584 & 1.405 & 4.752 & 0.002 & 2.702 & 1.468 & 4.976 & 0.001 \\
\hline NLR & & & & & & & & & & & & \\
\hline Low & 1 & & & & & & & & & & & \\
\hline High & 1.338 & 0.904 & 1.981 & 0.146 & & & & & & & & \\
\hline AAPR & & & & & & & & & & & & \\
\hline Low & 1 & & & & 1 & & & & & & & \\
\hline High & 0.679 & 0.482 & 0.956 & 0.027 & 0.649 & 0.459 & 0.918 & 0.015 & & & & \\
\hline Albumin & & & & & & & & & & & & \\
\hline Low & 1 & & & & & & & & 1 & & & \\
\hline High & 0.664 & 0.472 & 0.935 & 0.019 & & & & & 0.664 & 0.466 & 0.947 & 0.024 \\
\hline
\end{tabular}

Table 5. Prognostic models included AAPR and albumin for GBM patients in original cohort

\begin{tabular}{|c|c|c|c|c|c|c|c|c|}
\hline \multirow[t]{3}{*}{ Clinical variables } & \multicolumn{4}{|c|}{ Prognostic model (AAPR) } & \multicolumn{4}{|c|}{ Prognostic model (albumin) } \\
\hline & $\mathrm{HR}$ & $95 \% \mathrm{C}$ & & $\mathrm{p}$ value & HR & $95 \% \mathrm{C}$ & & $\mathrm{p}$ value \\
\hline & & LL & UL & & & LL & UL & \\
\hline \multicolumn{9}{|l|}{ Gender } \\
\hline Male & 1 & & & & 1 & & & \\
\hline Female & 0.649 & 0.466 & 0.904 & 0.011 & 0.662 & 0.475 & 0.922 & 0.015 \\
\hline \multicolumn{9}{|l|}{ Adjuvant therapy } \\
\hline Chemotherapy and radiotherapy & 1 & & & & 1 & & & \\
\hline Chemotherapy or radiotherapy & 2.763 & 1.852 & 4.122 & $<0.001$ & 2.972 & 1.985 & 4.452 & $<0.001$ \\
\hline None & 4.248 & 2.839 & 6.356 & $<0.001$ & 4.033 & 2.699 & 6.026 & $<0.001$ \\
\hline
\end{tabular}




\begin{tabular}{|c|c|c|c|c|c|c|c|c|}
\hline \multirow[t]{3}{*}{ Clinical variables } & \multicolumn{4}{|c|}{ Prognostic model (AAPR) } & \multicolumn{4}{|c|}{ Prognostic model (albumin) } \\
\hline & \multirow[t]{2}{*}{ HR } & \multicolumn{2}{|c|}{$95 \% \mathrm{CI}$} & \multirow[t]{2}{*}{$\mathrm{p}$ value } & \multirow[t]{2}{*}{ HR } & \multicolumn{2}{|l|}{$95 \% \mathrm{CI}$} & \multirow[t]{2}{*}{$\mathrm{p}$ value } \\
\hline & & LL & UL & & & LL & UL & \\
\hline \multicolumn{9}{|l|}{ Ki67 } \\
\hline$\geq 30 \%$ & 1 & & & & 1 & & & \\
\hline$<30 \%$ & 0.732 & 0.535 & 1.002 & 0.051 & 0.732 & 0.534 & 1.003 & 0.052 \\
\hline \multicolumn{9}{|l|}{ IDH-1 } \\
\hline Mutant & 1 & & & & 1 & & & \\
\hline Wildtype & 2.265 & 1.347 & 3.809 & 0.002 & 2.405 & 1.420 & 4.074 & 0.001 \\
\hline \multicolumn{9}{|l|}{ AAPR } \\
\hline Low & 1 & & & & & & & \\
\hline High & 0.589 & 0.430 & 0.807 & 0.001 & & & & \\
\hline \multicolumn{9}{|l|}{ Albumin } \\
\hline Low & & & & & 1 & & & \\
\hline High & & & & & 0.632 & 0.458 & 0.873 & 0.005 \\
\hline C-index & 0.721 & & & & 0.715 & & & \\
\hline AIC & 1452.24 & & & & 1455.95 & & & \\
\hline
\end{tabular}

Significant findings are expressed in bold and italic.

Abbreviations: HR, hazard ratio; CI, confidence interval; LL, lower limit; UL, upper limit; IDH-1, Isocitrate dehydrogenase-1; AAPR, albumin to alkaline phosphatase ratio; GBM, glioblastoma.

Table 6. Prognostic models included AAPR and albumin for GBM patients in PSM cohort

\begin{tabular}{|c|c|c|c|c|c|c|c|c|}
\hline \multirow[t]{3}{*}{ Clinical variables } & \multicolumn{4}{|c|}{ Prognostic model (AAPR) } & \multicolumn{4}{|c|}{ Prognostic model (Albumin) } \\
\hline & \multirow[t]{2}{*}{ HR } & \multicolumn{2}{|c|}{$95 \% \mathrm{CI}$} & \multirow[t]{2}{*}{$\mathrm{p}$ value } & \multirow[t]{2}{*}{ HR } & \multicolumn{2}{|c|}{$95 \%$ CI } & \multirow[t]{2}{*}{$\mathrm{p}$ value } \\
\hline & & $\overline{\mathrm{LL}}$ & UL & & & $\overline{\mathrm{LL}}$ & UL & \\
\hline \multicolumn{9}{|l|}{ Adjuvant therapy } \\
\hline Chemotherapy and radiotherapy & 1 & & & & 1 & & & \\
\hline Chemotherapy or radiotherapy & 2.866 & 1.834 & 4.478 & $<0.001$ & 3.119 & 1.986 & 4.899 & $<0.001$ \\
\hline None & 4.062 & 2.605 & 6.335 & $<0.001$ & 3.754 & 2.410 & 5.849 & $<0.001$ \\
\hline \multicolumn{9}{|l|}{ IDH-1 } \\
\hline Mutant & 1 & & & & 1 & & & \\
\hline Wildtype & 2.584 & 1.405 & 4.752 & 0.002 & 2.702 & 1.468 & 4.976 & 0.001 \\
\hline \multicolumn{9}{|l|}{ AAPR } \\
\hline Low & 1 & & & & & & & \\
\hline High & 0.649 & 0.459 & 0.918 & 0.015 & & & & \\
\hline \multicolumn{9}{|l|}{ Albumin } \\
\hline Low & & & & & 1 & & & \\
\hline High & & & & & 1.505 & 1.056 & 2.145 & 0.024 \\
\hline C-index & 0.698 & & & & 0.695 & & & \\
\hline AIC & 1091.43 & & & & 1094.86 & & & \\
\hline
\end{tabular}

Significant findings are expressed in bold and italic.

Abbreviations: PSM, propensity score matching; HR, hazard ratio; CI, confidence interval; LL, lower limit; UL, upper limit; IDH-1, Isocitrate dehydrogenase-1; AAPR,

albumin to alkaline phosphatase ratio; GBM, glioblastoma.

The examinations and treatments of GBM are expensive and represent a significant economic burden on health care system all around the world [47]. Compare with liquid biopsy, molecular test, and advanced magnetic resonance imaging (MRI) examination, blood biomarkers such like AAPR, NLR, platelet to lymphocyte ratio (PLR), and lymphocyte to monocyte ratio (LMR) are convenient, cheap, and time-saving. These easily acquired parameters help us understand this disease in more comprehensive perspective and make an accurate judgment on diagnosis and treatment strategies in GBM patients.

There are still some limitations in the current study. At first, the sample size is relatively small so that results of the study could not be further validated using external validation methods. Secondly, postoperative AAPR should be included in the follow-up plan so that we can further evaluate the prognostic value throughout the course of GBM. Thirdly, progression of GBM could not be accurately assessed, some patients didn't even have a chance to be reexamined after operation, so we didn't include this part in our research. Fourthly, this research was just conducted by a single center, and multi-center collaboration should be reached to further verify the results.

\section{Conclusion}

To our knowledge, this is the first study focusing on validating the prognostic ability of preoperative AAPR in GBM. In the current study, AAPR is determined to be an independent risk factor of prognosis in patients with newly-diagnosed GBM, and its prognostic predictive ability is stronger than albumin. PSM analysis is also conducted to validate the results. Serum albumin and ALP are simple, 
affordable and relatively innocuous test that could serve as an objective prognostic parameter for GBM.

\section{Acknowledgements}

\section{Funding}

This study was supported by the Key Research and Development Item from the Department of Science and Technology of Sichuan Province, China (No. 2017SZ0006).

\section{Competing Interests}

The authors have declared that no competing interest exists.

\section{References}

1. Omuro A, DeAngelis LM. Glioblastoma and other malignant gliomas: a clinical review. Jama. 2013; 310: 1842-50.

2. Molinaro AM, Taylor JW, Wiencke JK, Wrensch MR. Genetic and molecular epidemiology of adult diffuse glioma. Nature reviews Neurology. 2019; 15: 405-17.

3. Bush NA, Chang SM, Berger MS. Current and future strategies for treatment of glioma. Neurosurgical review. 2017; 40: 1-14.

4. Louis DN, Perry A, Reifenberger G, von Deimling A, Figarella-Branger D, Cavenee WK, et al. The 2016 World Health Organization Classification of Tumors of the Central Nervous System: a summary. Acta neuropathologica. 2016; 131: 803-20.

5. Cloughesy TF, Cavenee WK, Mischel PS. Glioblastoma: from molecular pathology to targeted treatment. Annual review of pathology. 2014; 9: 1-25.

6. Balkwill F, Mantovani A. Inflammation and cancer: back to Virchow? Lancet (London, England). 2001; 357: 539-45.

7. Haemmerle M, Stone RL, Menter DG, Afshar-Kharghan V, Sood AK. The Platelet Lifeline to Cancer: Challenges and Opportunities. Cancer cell. 2018; 33: 965-83.

8. Swierczak A, Mouchemore KA, Hamilton JA, Anderson RL. Neutrophils: important contributors to tumor progression and metastasis. Cancer metastasis reviews. 2015; 34: 735-51

9. Diem S, Schmid S, Krapf M, Flatz L, Born D, Jochum W, et al. Neutrophil-to-Lymphocyte ratio (NLR) and Platelet-to-Lymphocyte ratio (PLR) as prognostic markers in patients with non-small cell lung cancer (NSCLC) treated with nivolumab. Lung cancer (Amsterdam, Netherlands). 2017; 111: 176-81.

10. Capone M, Giannarelli D, Mallardo D, Madonna G, Festino L, Grimaldi AM, et al. Baseline neutrophil-to-lymphocyte ratio (NLR) and derived NLR could predict overall survival in patients with advanced melanoma treated with nivolumab. 2018; 6: 74 .

11. Templeton AJ, McNamara MG, Šeruga B, Vera-Badillo FE, Aneja P, Ocaña A, et al. Prognostic role of neutrophil-to-lymphocyte ratio in solid tumors: a systematic review and meta-analysis. Journal of the National Cancer Institute. 2014; 106: dju124.

12. Djambas Khayat C, El Khorassani M, Lambert T, Gay V, Barthez-Toullec M, Lamazure J, et al. Clinical pharmacology, efficacy and safety study of a triple-secured fibrinogen concentrate in adults and adolescent patients with congenital fibrinogen deficiency. Journal of thrombosis and haemostasis : JTH. 2019; 17: 635-44.

13. Moujaess E, Fakhoury M, Assi T, Elias H, El Karak F, Ghosn M, et al. The Therapeutic use of human albumin in cancer patients' management. Critical reviews in oncology/hematology. 2017; 120: 203-9.

14. Schoppet M, Shanahan CM. Role for alkaline phosphatase as an inducer of vascular calcification in renal failure? Kidney international. 2008; 73: 989-91.

15. Horowitz MC, Bothwell AL, Hesslein DG, Pflugh DL, Schatz DG. B cells and osteoblast and osteoclast development. Immunological reviews. 2005; 208 : 141-53.

16. Shanmugham LN, Petrarca C, Castellani ML, Symeonidou I, Frydas S, Vecchiet J, et al. IL-1beta induces alkaline phosphatase in human phagocytes. Archives of medical research. 2007; 38: 39-44.

17. Haarhaus M, Brandenburg V, Kalantar-Zadeh K, Stenvinkel P, Magnusson P. Alkaline phosphatase: a novel treatment target for cardiovascular disease in CKD. Nature reviews Nephrology. 2017; 13: 429-42.

18. Chan AW, Chan SL. Albumin-to-alkaline phosphatase ratio: a novel prognostic index for hepatocellular carcinoma. 2015; 2015: 564057.

19. Tan $\mathrm{P}, \mathrm{Xie} \mathrm{N}, \mathrm{Ai} J, \mathrm{Xu} \mathrm{H}, \mathrm{Xu} \mathrm{H}$, Liu L, et al. The prognostic significance of Albumin-to-Alkaline Phosphatase Ratio in upper tract urothelial carcinoma. Scientific reports. 2018; 8: 12311.
20. Li D, Yu H, Li W. Albumin-to-alkaline phosphatase ratio at diagnosis predicts survival in patients with metastatic non-small-cell lung cancer. OncoTargets and therapy. 2019; 12: 5241-9.

21. Li X, Li B, Zeng H, Wang S, Sun X, Yu Y, et al. Prognostic value of dynamic albumin-to-alkaline phosphatase ratio in limited stage small-cell lung cancer. Future oncology (London, England). 2019; 15: 995-1006.

22. Kim JS, Keam B, Heo DS, Han DH, Rhee CS, Kim JH, et al. The Prognostic Value of Albumin-to-Alkaline Phosphatase Ratio before Radical Radiotherapy in Patients with Non-metastatic Nasopharyngeal Carcinoma: A Propensity Score Matching Analysis. Cancer research and treatment : official journal of Korean Cancer Association. 2019; 51: 1313-23.

23. Camp RL, Dolled-Filhart M, Rimm DL. X-tile: a new bio-informatics tool for biomarker assessment and outcome-based cut-point optimization. Clinical cancer research : an official journal of the American Association for Cancer Research. 2004; 10: 7252-9.

24. Xiong JP, Long JY, Xu WY, Bian J, Huang HC, Bai Y, et al. Albumin-to-alkaline phosphatase ratio: A novel prognostic index of overall survival in cholangiocarcinoma patients after surgery. World journal of gastrointestinal oncology. 2019; 11: 39-47.

25. Zhang K, Dong S, Jing YH, Gao HF, Chen LY, Hua YQ, et al. Albumin-toalkaline phosphatase ratio serves as a prognostic indicator in unresectable pancreatic ductal adenocarcinoma: a propensity score matching analysis. 2020; 20: 541 .

26. Gupta D, Lis CG. Pretreatment serum albumin as a predictor of cancer survival: a systematic review of the epidemiological literature. Nutrition journal. 2010; 9: 69 .

27. Fernández J, Clària J, Amorós A, Aguilar F, Castro M, Casulleras M, et al. Effects of Albumin Treatment on Systemic and Portal Hemodynamics and Systemic Inflammation in Patients With Decompensated Cirrhosis. Gastroenterology. 2019; 157: 149-62.

28. Kao HK, Löfstrand J, Loh CY, Lao WW, Yi JS, Chang YL, et al. Nomogram based on albumin and neutrophil-to-lymphocyte ratio for predicting the prognosis of patients with oral cavity squamous cell carcinoma. Scientific reports. 2018; 8: 13081

29. Miura K, Hamanaka K, Koizumi T, Kitaguchi Y, Terada Y, Nakamura D, et al. Clinical significance of preoperative serum albumin level for prognosis in surgically resected patients with non-small cell lung cancer: Comparative study of normal lung, emphysema, and pulmonary fibrosis. Lung cancer (Amsterdam, Netherlands). 2017; 111: 88-95.

30. Takeda K, Umezawa R, Takahashi N, Matsushita H, Kozumi M, Ishikawa Y, et al. Impact of change in serum albumin level during and after chemoradiotherapy in patients with locally advanced esophageal cancer. Esophagus : official journal of the Japan Esophageal Society. 2018; 15: 190-7.

31. Fan H, Shao ZY, Xiao YY, Xie ZH, Chen W, Xie H, et al. Comparison of the Glasgow Prognostic Score (GPS) and the modified Glasgow Prognostic Score (mGPS) in evaluating the prognosis of patients with operable and inoperable non-small cell lung cancer. Journal of cancer research and clinical oncology. 2016; 142: 1285-97.

32. Kuroda D, Sawayama H, Kurashige J, Iwatsuki M, Eto T, Tokunaga R, et al. Controlling Nutritional Status (CONUT) score is a prognostic marker for gastric cancer patients after curative resection. 2018; 21: 204-12.

33. Ren HY, Sun LL, Li HY, Ye ZM. Prognostic Significance of Serum Alkaline Phosphatase Level in Osteosarcoma: A Meta-Analysis of Published Data. BioMed research international. 2015; 2015: 160835.

34. Xu XS, Wan Y, Song SD, Chen W, Miao RC, Zhou YY, et al. Model based on $\gamma$-glutamyltransferase and alkaline phosphatase for hepatocellular carcinoma prognosis. World journal of gastroenterology. 2014; 20: 10944-52.

35. Rao SR, Snaith AE, Marino D, Cheng X, Lwin ST, Orriss IR, et al. Tumour-derived alkaline phosphatase regulates tumour growth, epithelial plasticity and disease-free survival in metastatic prostate cancer. British journal of cancer. 2017; 116: 227-36.

36. Hung HY, Chen JS, Chien Y, Tang R, Hsieh PS, Wen S, et al. Preoperative alkaline phosphatase elevation was associated with poor survival in colorectal cancer patients. International journal of colorectal disease. 2017; 32: 1775-8.

37. Karhade AV, Thio $\mathrm{Q}$ Kuverji $\mathrm{M}$, Ogink PT, Ferrone ML, Schwab JH. Prognostic value of serum alkaline phosphatase in spinal metastatic disease. British journal of cancer. 2019; 120: 640-6.

38. Ji F, Fu SJ, Guo ZY, Pang H, Ju WQ, Wang DP, et al. Prognostic value of combined preoperative lactate dehydrogenase and alkaline phosphatase levels in patients with resectable pancreatic ductal adenocarcinoma. Medicine. 2016; 95: e4065.

39. Mochizuki T, Kawahara T. The neutrophil-to-lymphocyte ratio (NLR) predicts adrenocortical carcinoma and is correlated with the prognosis. 2017; 17: 49.

40. Miyamoto R, Inagawa S, Sano N, Tadano S, Adachi S, Yamamoto M. The neutrophil-to-lymphocyte ratio (NLR) predicts short-term and long-term outcomes in gastric cancer patients. European journal of surgical oncology : the journal of the European Society of Surgical Oncology and the British Association of Surgical Oncology. 2018; 44: 607-12.

41. Han S, Liu Y, Li Q, Li Z, Hou H, Wu A. Pre-treatment neutrophil-tolymphocyte ratio is associated with neutrophil and T-cell infiltration and predicts clinical outcome in patients with glioblastoma. BMC cancer. 2015; 15: 617.

42. Zhang ZY, Zhan YB, Zhang FJ, Yu B, Ji YC, Zhou JQ, et al. Prognostic value of preoperative hematological markers combined with molecular pathology in patients with diffuse gliomas. Aging. 2019; 11: 6252-72. 
43. Bambury RM, Teo MY, Power DG, Yusuf A, Murray S, Battley JE, et al. The association of pre-treatment neutrophil to lymphocyte ratio with overall survival in patients with glioblastoma multiforme. Journal of neuro-oncology. 2013; 114: 149-54.

44. Lv Y, Zhang S, Liu Z, Tian Y, Liang N, Zhang J. Prognostic value of preoperative neutrophil to lymphocyte ratio is superior to systemic immune inflammation index for survival in patients with Glioblastoma. Clinical neurology and neurosurgery. 2019; 181: 24-7.

45. Brenner A, Friger M, Geffen DB, Kaisman-Elbaz T, Lavrenkov K. The Prognostic Value of the Pretreatment Neutrophil/Lymphocyte Ratio in Patients with Glioblastoma Multiforme Brain Tumors: A Retrospective Cohort Study of Patients Treated with Combined Modality Surgery, Radiation Therapy, and Temozolomide Chemotherapy. Oncology. 2019; 97: 255-63.

46. Lopes M, Carvalho B, Vaz R, Linhares P. Influence of neutrophil-lymphocyte ratio in prognosis of glioblastoma multiforme. Journal of neuro-oncology. 2018; 136: 173-80.

47. Waschke A, Arefian H, Walter J, Hartmann M, Maschmann J, Kalff R. Cost-effectiveness of the long-term use of temozolomide for treating newly diagnosed glioblastoma in Germany. Journal of neuro-oncology. 2018; 138: 359-67. 